\title{
General minimum Euclidean distance-based precoder for MIMO wireless systems
}

\author{
Quoc-Tuong Ngo*, Olivier Berder and Pascal Scalart
}

\begin{abstract}
In this article, we investigate the linear precoder based on the maximization of the minimum Euclidean distance between two received data vectors. This new precoding matrix is expressed as the product of a power allocation matrix and an input-shaping matrix. The input-shaping matrix is selected as a normalized discrete Fourier transform-matrix, and the optimal power allocation depends on the channel characteristics. For each number of available datastreams, we propose a general form of the optimized precoding matrix. These forms are suitable for different transmit channels and especially for all rectangular quadrature amplitude modulation modulations. We show, in the simulation results, that the proposed precoder provides a significant improvement in terms of bit error rate performance compared to other traditional precoding strategies.
\end{abstract}

Keywords: MIMO, CSIT, Linear precoder, Spatial multiplexing, ML receiver, Minimum Euclidean distance

\section{Introduction}

In wireless communication, the systems that employ multiple antennas at both the transmitter and the receiver known as multiple-input multiple-output (MIMO) systems, not only offer the diversity and capacity gains, but also achieve higher link reliability in comparison with single antenna systems [1]. The idea of using multiple transceivers and receivers was first proposed by Bell Lab [2], and, then, has been utilized worldwide to adapt to various high-speed wireless transmissions.

In order to overcome the multi-path effect and improve the robustness of MIMO systems, a linear precoding transceiver can be used. Precoding is a processing technique that exploits the channel state information at transmitter (CSIT) by operating on the signal before transmission [3]. In fact, the transmitted vectors are premultiplied by a precoding matrix, which adapts to various forms of the channel knowledge. Various optimization criteria can be used to design a precoding matrix such as maximizing the output capacity [4], minimizing the bit error rate (BER) [5], maximizing the received signal-tonoise ratio (SNR) [6], minimizing the mean square error (MSE) [7], and maximizing the minimum singular value [8]. These precoders belong to an important set of linear

*Correspondence: qngo@irisa.fr

IRISA, University of Rennes 1, Rennes, France precoding techniques called as diagonal precoders. A specific precoding matrix, which follows the non-diagonal structure, was proposed in [9]. This design employ Schurconvex functions in order to optimize MSE-based or BER-based criteria.

In this article, we consider another non-diagonal linear precoder that optimizes the minimum Euclidean distance $\left(\max -d_{\min }\right)$ between two received data vectors. This precoder improves the BER performance of the MIMO systems, especially when an ML detection is used at the receiver. It is because that the minimum distance is mutual information optimal for discrete input at hight SNR [10]. The optimal solution of $\max -d_{\min }$ precoder is proposed in $[11,12]$ for two transmit datastreams with 4-QAM and 16-QAM modulations. By decomposing the channel into $2 \times 2$ eigen-channel matrices and optimizing the distance $d_{\text {min }}$ for each sub-system, Vrigneau et al. [13] proposed a sub-optimal precoder for large MIMO channels. However, this solution is also available for low-order Quadrature Amplitude Modulation (QAM) modulations. It is because the optimized solution for two-datastreams transmission depends on the symbol alphabet, the detection rule, and the characteristic of the transmit channel. Another suboptimal design of the max $-d_{\min }$ precoder, which allows transmitting more than two independent datastreams and

\section{望 Springer}

(c) 2013 Ngo et al: licensee Springer. This is an Open Access article distributed under the terms of the Creative Commons Attribution License (http://creativecommons.org/licenses/by/2.0), which permits unrestricted use, distribution, and reproduction in any medium, provided the original work is properly cited. 
increasing the order of the modulations, is presented in [14]. But, the precoding scheme considers just a blockToeplitz form of the channel matrix and, therefore, is only suitable for quasi-stationary MIMO channels. In addition, we can apply lattice invariant operations with the linear precoding in order to transform the transmit channel into a lattice generator matrix with large minimum distance separation [15].

The problem of high-order QAM modulations and the number of datastreams for minimum distance-based precoder was settled in this article. The precoding matrix is then factorized as the product of a diagonal power allocation matrix and an input-shaping matrix. The inputshaping matrix is selected as a discrete Fourier transform (DFT) matrix, and the power allocation matrix varies depending on the channel characteristics. At that time, the expression of the precoding matrix is less complex with only $b$ variables corresponding to the $b$ diagonal entries of the power allocation matrix. The idea of using the DFTbased matrix in precoding scheme was also proposed in [16], but this precoding design is only due to the power leakage suppression.

We propose, herein, a sub-optimal DFT-based precoding scheme which not only reduces the complexity, but also improves the minimum distance. A numerical approach is considered to indicate which difference vectors provide the minimum distances, and by equalizing these distances, it is possible to obtain the optimized precoding matrix. For each number of available datastreams, we propose a general precoding matrix for all rectangular QAM modulations. The simulation results confirm a significant BER improvement of our new precoder in comparison with other traditional precoding strategies.

The remainder of this article is organized as follows. Section "System overview" presents a brief introduction of virtual MIMO channel representations and linear precoding systems. The new parameterized form of the precoding matrix is described in "Parameterization of the precoding matrix". Section "Design of the precoding matrix" is devoted to the description of the new precoder which is based on the observation of the SNR-like matrix. In Section "Optimized precoder for rectangular QAM modulations", we propose general extensions of the precoder for large MIMO channels and all rectangular QAM modulations. Simulation results in comparison with other traditional precoders are presented in section "Simulation results". Finally, the article ends with "Conclusion" section.

\section{System overview}

We consider a MIMO system with $n_{T}$ transmit and $n_{R}$ receive antennas. For each Rayleigh fading channel used, $b$ independent datastreams are transmitted, with $b \leq \operatorname{rank}(\mathbf{H}) \leq \min \left(n_{T}, n_{R}\right)$. The received signal is expressed as

$$
\mathbf{y}=\mathbf{G H F s}+\mathbf{G} \eta
$$

where $\mathbf{y}$ is the $b \times 1$ received symbols vector, $\mathbf{s}$ is the $b \times 1$ transmitted symbols vector, $\eta$ is the $n_{R} \times 1$ additive Gaussian noise vector, $\mathbf{H}$ is the $n_{R} \times n_{T}$ channel matrix, $\mathbf{F}$ is the $n_{T} \times b$ precoding matrix, and $\mathbf{G}$ is the $b \times n_{R}$ decoding matrix.

When full channel state information (CSI) is available at both transmitter and receiver, the channel can be full-rank diagonalized by using a successive linear transformations presented in [11]. The precoding and decoding matrices are then decomposed as $\mathbf{F}=\mathbf{F}_{\nu} \mathbf{F}_{d}$ and $\mathbf{G}=\mathbf{G}_{d} \mathbf{G}_{\nu}$. In which, the couple $\left(\mathbf{F}_{d}, \mathbf{G}_{d}\right)$ is used to optimize the minimum distance, while $\left(\mathbf{F}_{v}, \mathbf{G}_{v}\right)$ is needed to diagonalize the transmit channel. The MIMO channel representation is therefore defined by

$$
\mathbf{y}=\mathbf{G}_{d} \mathbf{H}_{v} \mathbf{F}_{d} \mathbf{s}+\mathbf{G}_{d} \boldsymbol{\eta}_{v}
$$

where $\mathbf{H}_{v}$ is the $b \times b$ virtual channel matrix, $\eta_{v}=\mathbf{G}_{v} \eta$ is the $b \times 1$ transformed additive Gaussian noise vector. One should note that the virtual channel matrix $\mathbf{H}_{v}$ is now diagonal and defined by

$$
\mathbf{H}_{v}=\operatorname{diag}\left(\sqrt{\rho_{1}}, \ldots, \sqrt{\rho_{b}}\right),
$$

where $\rho_{i}$ stands for every subchannel gain and is sorted in decreasing order.

In this article, an ML detection is considered at the receiver, and then, the decoding matrix $\mathbf{G}_{d}$ has no effect on the performance. Hence, $\mathbf{G}_{d}$ is consequently assumed to be an identity matrix of size $b$. The virtual system model can be then simplified as

$$
\mathbf{y}=\mathbf{H}_{v} \mathbf{F}_{d} \mathbf{s}+\boldsymbol{\eta}_{v}
$$

The precoding matrix $\mathbf{F}_{d}$ is designed under the power constraint

$$
\operatorname{trace}\left\{\mathbf{F}_{d} \mathbf{F}_{d}^{*}\right\}=E_{s},
$$

where $E_{s}$ is the average transmit power.

\section{Parameterization of the precoding matrix}

We now design a precoding matrix to improve the probability of error subject to the constraint of transmission powers. This design is difficult because it is rarely solvable in closed form: the solution depends on the symbol alphabet, the number of parallel datastreams, and channel characteristics. In general, the average error probability can be approximated by [17]

$$
P_{e} \simeq \frac{1}{M} \sum_{i=1}^{M} \sum_{\substack{j=1 \\ j \neq i}}^{M} Q\left(\frac{\bar{d}_{i j}}{2 \sqrt{N_{0}}} \times \sqrt{E_{s}}\right),
$$


where $N_{0}$ is the variance of the white Gaussian noise $\eta_{v}$, and $\bar{d}_{i j}$ is the normalized Euclidean distance between two vector $\mathbf{s}_{i}$ and $\mathbf{s}_{j}$ at the receiver. Let us note $N_{i}$ the number of distances $\bar{d}_{i j}$ such that $\bar{d}_{i j}=d_{\text {min }}$, where $d_{\text {min }}$ denotes the minimum Euclidean distance and is defined by

$$
d_{\min }^{2}=\min _{\mathbf{s}_{k}, \mathbf{s}_{l} \in S, \mathbf{s}_{k} \neq \mathbf{s}_{l}}\left\|\mathbf{H}_{v} \mathbf{F}_{d}\left(\mathbf{s}_{k}-\mathbf{s}_{l}\right)\right\|^{2} .
$$

The probability of error in (6) can be now simplified as

$$
\begin{aligned}
P_{e} & \approx \frac{1}{M} \sum_{i=1}^{M} \mathrm{~N}_{i} Q\left(\frac{\bar{d}_{\text {min }}}{2 \sqrt{N_{0}}} \times \sqrt{E_{s}}\right) \\
& \approx \mathrm{N}_{d_{\min }} Q\left(\frac{\bar{d}_{\text {min }}}{2 \sqrt{N_{0}}} \times \sqrt{E_{s}}\right),
\end{aligned}
$$

where $M$ is the number of all possible transmitted vectors $\mathbf{s}$, and $N_{d_{\min }}=\frac{1}{M} \sum_{i=1}^{M} N_{i}$. It is observed that when an ML detection is considered at the receiver, a key to reduce the probability of error is maximizing the minimum Euclidean distance between received symbols. We can now formulate the design problem as follows

$$
\underset{\mathbf{F}_{d}}{\operatorname{argmax}} d_{\min }^{2}
$$

subject to: $\operatorname{trace}\left\{\mathbf{F}_{d} \mathbf{F}_{d}^{*}\right\}=E_{s}$.

By using a singular value decomposition, a linear precoder can be considered as a combination of an input shaper and a multimode beamformer with per-beam power allocation [3]

$$
\mathbf{F}_{d}=\mathbf{A} \Sigma \mathbf{B}^{*},
$$

where $\mathbf{A}$ and $\mathbf{B}^{*}$ are $b \times b$ unitary matrices, and $\boldsymbol{\Sigma}$ is a diagonal matrix. The orthogonal beam directions are the left singular matrix $\mathbf{A}$, of which each column represents a beam direction (pattern). It is noted that the matrix A contains all eigenvectors of the matrix $\mathbf{F}_{d} \mathbf{F}_{d}^{*}$, thus it is often referred to as eigen-beamforming. The matrix $\boldsymbol{\Sigma}$ controls the power allocation on each beam. These powers correspond to the squared singular values of $\Sigma^{2}$. The right singular matrix $\mathbf{B}^{*}$ concerns with the rotation and scaling of the input symbols on each beam and hence is referred to as the input-shaping matrix.

Let us define $\breve{\mathbf{x}}$ a difference vector as $\breve{\mathbf{x}}=\mathbf{s}_{k}-\mathbf{s}_{l}$, with $\mathbf{s}_{k} \neq \mathbf{s}_{l}$, and the set which contains all possible difference vectors as $\breve{X}$. The optimized criterion is then

$$
\begin{aligned}
d_{\min }^{2} & =\min _{\breve{\mathbf{x}} \in \breve{X}}\left\|\mathbf{H}_{\nu} \mathbf{F}_{d} \breve{\mathbf{x}}\right\|^{2} \\
& =\min _{\breve{\mathbf{x}} \in \breve{X}} \breve{\mathbf{x}}^{*} \mathbf{F}_{d}^{*} \mathbf{H}_{\nu}^{*} \mathbf{H}_{\nu} \mathbf{F}_{d} \breve{\mathbf{x}} \\
& =\min _{\breve{\mathbf{x}} \in \breve{X}} \breve{\mathbf{x}}^{*} \mathbf{B} \boldsymbol{\Sigma}^{*} \mathbf{A}^{*} \mathbf{R}_{H} \mathbf{A} \boldsymbol{\Sigma} \mathbf{B}^{*} \breve{\mathbf{x}},
\end{aligned}
$$

where $\mathbf{R}_{H}$ denotes the channel covariance matrix and is given by $\mathbf{R}_{H}=\mathbf{H}_{v}^{*} \mathbf{H}_{v}=\operatorname{diag}\left(\rho_{1}, \ldots, \rho_{b}\right)$. One should note that $\mathbf{R}_{H}$ is a diagonal matrix since the virtual channel $\mathbf{H}_{v}$ is already diagonalized.

Lemma 1. Without loss of optimality, the left singular matrix $\mathbf{A}$ of the optimal precoder $\mathbf{F}_{d}$ can always be chosen to coincide with an identity matrix.

Proof. See in [15].

From the result in Lemma 1, it follows that the max $-d_{\text {min }}$ precoder can be parameterized as

$$
\mathbf{F}_{d}=\mathbf{\Sigma} \mathbf{B}^{*}
$$

where $\mathbf{B}^{*}$ is a $b \times b$ unitary matrix, and $\boldsymbol{\Sigma}=$ $\operatorname{diag}\left(\sqrt{\sigma_{1}}, \ldots, \sqrt{\sigma_{b}}\right)$ is a $b \times b$ diagonal matrix with nonnegative real numbers on the diagonal. The power constraint in (5) can be then rewritten as

$$
\operatorname{trace}\left\{\mathbf{F}_{d} \mathbf{F}_{d}^{*}\right\}=\operatorname{trace}\left\{\boldsymbol{\Sigma} \boldsymbol{\Sigma}^{*}\right\}=E_{s} .
$$

\section{Design of the precoding matrix}

\section{Principle of the approach}

Design optimizing the minimum Euclidean distance is difficult to deal with because of two reasons. First, the space of solution is large and exponentially proportional to the number of datastreams $b$. Second, the exact expression of $\max -d_{\text {min }}$ precoder depends on many parameters such as the symbol alphabet or the characteristic of the virtual channel. Here, we propose a design that can come close to the desired goal. Based on (8), the formulation of the problem can be rewritten as

$$
\max _{\mathbf{F}_{d}} \min _{\breve{\mathbf{x}} \in \breve{X}} d_{\breve{\mathbf{x}}}^{2}=\breve{\mathbf{x}}^{*} \mathbf{F}_{d}^{*} \mathbf{H}_{v}^{*} \mathbf{H}_{v} \mathbf{F}_{d} \breve{\mathbf{x}}
$$

Let us define an SNR-like matrix of $\mathbf{F}_{d}$ as $\operatorname{SNR}\left(\mathbf{F}_{d}\right)=$ $\mathbf{F}_{d}^{*} \mathbf{H}_{v}^{*} \mathbf{H}_{v} \mathbf{F}_{d}$. Instead of optimizing (13), we can obtain a suboptimal but more general solution by realizing some properties of $\operatorname{SNR}\left(\mathbf{F}_{d}\right)$. Scaglione et al. [8] proposed a suboptimal precoder which is based on the observation of the minimum eigenvalue of $\operatorname{SNR}\left(\mathbf{F}_{d}\right)$. We present, herein, another suboptimal solution that considers the minimum diagonal element of the SNR-like matrix. Let us denote the diagonal elements of $\operatorname{SNR}\left(\mathbf{F}_{d}\right)$ as $\boldsymbol{\delta}_{k}$, we have

$$
d_{\breve{\mathbf{x}}}^{2}=\breve{\mathbf{x}}^{*} \operatorname{SNR}\left(\mathbf{F}_{d}\right) \breve{\mathbf{x}}=\sum_{i=1}^{b} \delta_{i} x_{i}^{2}+\mathcal{O}\left(x_{i} x_{j}\right)_{x_{i} \neq x_{j}},
$$

with $\breve{\mathbf{x}}=\left[x_{1}, \ldots, x_{b}\right]^{T}$. In order to simplify the complexity of the solution, we assume that the function $\mathcal{O}\left(x_{i} x_{j}\right)$ has little influence on the performance. Then, the design problem can be simplified as

$$
\max _{\mathbf{F}_{d}} \min _{\breve{\mathbf{x}} \in \breve{X}} \sum_{i=1}^{b} \delta_{i} x_{i}^{2} .
$$


The criterion on the right-hand side of (15) has a lower bound

$$
\min _{\breve{\mathbf{x}} \in \breve{X}} \sum_{i=1}^{b} \delta_{i} x_{i}^{2} \geq \delta_{\min } \min _{\breve{\mathbf{x}} \in \breve{X}} \sum_{i=1}^{b} x_{i}^{2}=\delta_{\min } \min _{\breve{\mathbf{x}} \in \breve{X}}\|\breve{\mathbf{x}}\|^{2},
$$

where $\delta_{\text {min }}$ denotes the minimum diagonal element of $\operatorname{SNR}\left(\mathbf{F}_{d}\right)$. It is observed that increasing the minimum diagonal element $\delta_{\min }\left(\operatorname{SNR}\left(\mathbf{F}_{d}\right)\right)$ to higher value will possibly obtain a suboptimal solution of the minimum distance criterion. Therefore, we first deal with $\delta_{\min }\left(\operatorname{SNR}\left(\mathbf{F}_{d}\right)\right)$ and then maximize its value. By substituting (11) into the form of $\operatorname{SNR}\left(\mathbf{F}_{d}\right)$, we obtain

$$
\operatorname{SNR}\left(\mathbf{F}_{d}\right)=\mathbf{B} \boldsymbol{\Sigma}^{*} \mathbf{H}_{v}^{*} \mathbf{H}_{v} \boldsymbol{\Sigma} \mathbf{B}^{*}=\mathbf{B} \Upsilon \mathbf{B}^{*}
$$

where $\Upsilon=\operatorname{diag}\left(\rho_{1} \sigma_{1}, \ldots, \rho_{b} \sigma_{b}\right)=\operatorname{diag}\left(\lambda_{1}, \ldots, \lambda_{b}\right)$ is a diagonal matrix with non-negative real numbers on the diagonal. For any given $\boldsymbol{\Upsilon}$, we first consider an optimal choice of the unitary matrix $\mathbf{B}$ which maximizes the minimum diagonal element of $\operatorname{SNR}\left(\mathbf{F}_{d}\right)$. Such a matrix $\mathbf{B}$ is provided by the following lemma.

Lemma 2. Given a $b \times b$ diagonal matrix $\Upsilon$ whose diagonal elements are non-negative and a unitary matrix $\mathbf{B}$ of size $b$, then we have the following properties

1.

$$
\max _{\mathbf{B} \mathbf{B}^{*}=\mathbf{I}_{b}} \min _{i}\left[\mathbf{B} \Upsilon \mathbf{B}^{*}\right]_{i, i}=\frac{\operatorname{trace}(\boldsymbol{\Upsilon})}{b} .
$$

2. The optimized value in (18) is provided by a normalized DFT-matrix

$$
\mathbf{B}^{*}=\mathbf{D}_{b}=\frac{1}{\sqrt{b}}\left(\begin{array}{ccccc}
1 & 1 & 1 & \ldots & 1 \\
1 & \omega & \omega^{2} & \ldots & \omega^{b-1} \\
1 & \omega^{2} & \omega^{4} & \ldots & \omega^{2(b-1)} \\
\vdots & \vdots & \vdots & & \vdots \\
1 & \omega^{b-1} & \omega^{2(b-1)} & \ldots & \omega^{(b-1)(b-1)}
\end{array}\right) \text {, }
$$

where $\omega$ is a primitive $b$ th root of unity, i.e., $\omega=e^{-\frac{2 \pi i}{b}}$.

Proof. First, we prove that the right-hand side of (18) is the upper-bound for the left-hand side. Then, we show that the DFT-matrix $\mathbf{D}_{b}$ can provide this upper bound.

1. Since $\mathbf{B}$ is a unitary matrix and $\boldsymbol{\Upsilon}$ is a diagonal matrix, we have

$$
\sum_{i=1}^{b} \delta_{i}=\operatorname{trace}\left(\mathbf{B} \Upsilon \mathbf{B} \mathbf{B}^{*}\right)=\operatorname{trace}(\mathbf{\Upsilon})
$$

Furthermore, since the diagonal elements of $\boldsymbol{\Upsilon}$ are non-negative, those of $\mathbf{B} \boldsymbol{\Upsilon} \mathbf{B}^{*}$ are non-negative, too. Given the set of $b$ non-negative numbers $\left\{\alpha_{i}\right\}_{i=1}^{b}$ that sum to $\mathcal{M}$, the minimum number is obviously less than $\mathcal{M} / b$. The left-hand side of (18) is, therefore, upper-bounded by

$$
\min _{i}\left[\mathbf{B} \Upsilon \mathbf{B}^{*}\right]_{i, i} \leq \frac{\sum_{i=1}^{b} \delta_{i}}{b}=\frac{\operatorname{trace}(\boldsymbol{\Upsilon})}{b}
$$

2. Let us define $\beta_{i, j}$ is the $(i, j)$ element of the matrix $\mathbf{B}^{*}$, we have

$$
\left[\mathbf{B} \Upsilon \mathbf{B}^{*}\right]_{i, i}=\sum_{j=1}^{b} \lambda_{j}\left\|\beta_{i, j}\right\|^{2}
$$

If $\mathbf{B}^{*}$ is selected as a DFT-matrix, i.e., the magnitude of each element of the DFT-matrix $\mathbf{D}_{b}$ is equal to $\left|\beta_{i, j}\right|^{2}=1 / b$, we obtain that

$$
\left[\mathbf{B} \Upsilon \mathbf{B}^{*}\right]_{i, i}=\sum_{j=1}^{b} \lambda_{j} \frac{1}{b}=\frac{\operatorname{trace}(\boldsymbol{\Upsilon})}{b}
$$

for all $1 \leq i \leq b$.

Lemma provides the key to obtain a sub-optimal solution for the problem of maximizing the minimum distance. One should note that the minimum Euclidean distances on the received constellation are always provided by some difference vectors. By equalizing these difference distances, we can obtain an analytical solution of the precoding matrix.

Proposition 1. In order to equalize any difference distances, we can retain the input-shaping matrix $\mathbf{B}^{*}$, and change only the power allocation matrix $\boldsymbol{\Sigma}$.

Proof. We assume that, at the channel $\hat{\mathbf{H}}_{\nu} \operatorname{diag}$ $\left(\sqrt{\hat{\rho}_{1}}, \ldots, \sqrt{\hat{\rho}_{b}}\right)$ and $\hat{\boldsymbol{\Sigma}}=\operatorname{diag}\left(\sqrt{\hat{\sigma}_{1}}, \ldots, \sqrt{\hat{\sigma}_{b}}\right)$, two difference vectors $\breve{\mathbf{x}}_{1}, \breve{\mathbf{x}}_{2}$ have the same Euclidean distances

$$
\left\{\begin{array}{l}
d_{\breve{\mathbf{x}}_{1} \mid \hat{\mathbf{H}}_{v}}^{2}=\left\|\hat{\mathbf{H}}_{v} \hat{\boldsymbol{\Sigma}} \mathbf{B} \breve{\mathbf{x}}_{1}\right\|^{2} \\
d_{\breve{\mathbf{x}}_{2} \mid \hat{\mathbf{H}}_{v}}^{2}=\left\|\hat{\mathbf{H}}_{v} \hat{\boldsymbol{\Sigma}} \mathbf{B} \breve{\mathbf{x}}_{2}\right\|^{2}
\end{array}\right.
$$

When the channel varies from $\hat{\mathbf{H}}_{v}$ to $\mathbf{H}_{v}=$ $\operatorname{diag}\left(\sqrt{\rho_{1}}, \ldots, \sqrt{\rho_{b}}\right)$, let us define a diagonal matrix $\boldsymbol{\Sigma}$ with real non-negative elements such that

$$
\sigma_{i} \rho_{i}=\kappa \hat{\sigma}_{i} \hat{\rho}_{i}
$$


where $\kappa$ is a constant. By substituting $\hat{\sigma}_{i}$ into the power constraint in (12), we obtain

$$
\operatorname{trace}\left\{\Sigma \Sigma^{*}\right\}=\sum_{i=1}^{b} \sigma_{i}=\kappa \sum_{i=1}^{b} \hat{\sigma}_{i}\left(\frac{\hat{\rho}_{i}}{\rho_{i}}\right)=E_{s}
$$

or

$$
\kappa=\frac{E_{s}}{\sum_{i=1}^{b} \hat{\sigma}_{i} \hat{\rho}_{i} / \rho_{i}} .
$$

The Euclidean distance provided by $\breve{\mathbf{x}}_{1}$ is then

$$
\begin{aligned}
d_{\breve{\mathbf{x}}_{1} \mid \mathbf{H}_{v}}^{2} & =\left\|\mathbf{H}_{v} \boldsymbol{\Sigma} \mathbf{B} \breve{\mathbf{x}}_{1}\right\|^{2} \\
& =\left\|\sqrt{\kappa} \hat{\mathbf{H}}_{v} \hat{\mathbf{\Sigma}} \mathbf{B} \breve{\mathbf{x}}_{1}\right\|^{2} \\
& =\kappa d_{\breve{\mathbf{x}}_{1} \mid \hat{\mathbf{H}}_{v}}^{2} .
\end{aligned}
$$

Similarly, we get

$$
d_{\breve{\mathbf{x}}_{2} \mid \mathbf{H}_{v}}^{2}=\kappa d_{\breve{\mathbf{x}}_{2} \mid \hat{\mathbf{H}}_{v}}^{2} .
$$

Since $d_{\breve{\mathbf{x}}_{1} \mid \hat{\mathbf{H}}_{v}}^{2}=d_{\breve{\mathbf{x}}_{2} \mid \hat{\mathbf{H}}_{v}}^{2}$, we obtain $d_{\breve{\mathbf{x}}_{1} \mid \mathbf{H}_{v}}^{2}=d_{\breve{\mathbf{x}}_{2} \mid \mathbf{H}_{v}}^{2}$. It is obvious that $\kappa$ does not depend on the difference vectors $\breve{\mathbf{x}}_{1}$ and $\breve{\mathbf{x}}_{2}$. It means that for any number of difference vectors, we can equalize their difference distances by changing only the power allocation matrix $\Sigma$.

\section{Proposed model}

Now, we present the key to design a new linear precoder. First, the precoding matrix $\mathbf{F}_{d}$ is factorized as the product of the power allocation matrix $\boldsymbol{\Sigma}$ and the scaling matrix $\mathbf{B}^{*}$.

Beside the role of controlling the power allocation on each stream, the matrix $\boldsymbol{\Sigma}$ also determines how many virtual channels used to transmit signal. One should note the maximum number of activate virtual channels is upperbounded by the rank of matrix $\mathbf{H}$. In other words, the non-null diagonal elements of $\boldsymbol{\Sigma}$ are less than the number of datastreams $b$. We assume that the signal is transmitted on $k$ subchannels, i.e., $k \leq b=\operatorname{rank}(\mathbf{H})$. The matrix $\mathbf{B}^{*}$ is then selected as a normalized DFT-matrix of size $k$. According to Proposition 1, the diagonal matrix $\boldsymbol{\Sigma}$ depends on the channel characteristics, and has $k$ positive real elements on the diagonal. We have total $b$ different expressions of the precoding matrix $\mathbf{F}_{d}$ corresponding to $b$ precoders which pour powers on $1,2, \ldots$, and $b$ virtual subchannels.

The precoding system structure, which contains an input-shaping matrix and a power allocation matrix, is shown in Figure 1. Due to different forms of CSIT, the precoder first decides number of virtual subchannels used for transmission, and then maps the data-bits into $k$ symbols. The method used for selecting the modulation will be discussed in Section "Range of definition". After that these symbols are pre-processed by a DFT block of size $k$. At the end of the precoder, the transmit signal is directly operated by a power distribution block, i.e., multiplied to a diagonal matrix $\boldsymbol{\Sigma}$.

The expression of the power allocation matrix $\boldsymbol{\Sigma}$ depends on the symbol alphabet and the modulation used at the transmitter. In the following section, we propose a simple solution for one of the most common schemes: rectangular QAM.

\section{Optimized precoder for rectangular QAM modulations \\ Expressions of the precoding matrix}

For a rectangular $4^{m}$-QAM modulation, the transmit symbols belong to the set

$$
S=\frac{1}{\sqrt{M_{s}}}\{a+b i ; a-b i ;-a+b i ;-a-b i\},
$$

where $M_{s}=\frac{2}{3}\left(4^{m}-1\right)$ and $a, b \in\left\{1,3, \ldots, 2^{m}-1\right\}$.

In the new precoding scheme, the input-shaping matrix $\mathbf{B}^{*}$ is given by a DFT-matrix of size $b$. Our objective becomes to determine the matrix $\boldsymbol{\Sigma}$ subject to the power constraint (12) in order to improve the minimum distance performance. To derive the analytical solution of the power allocation matrix $\Sigma$ in (11), we have to follow three main steps:

(i) Eliminate the collinear difference vectors $\breve{\mathbf{x}} \in \breve{X}$ in order to reduce the space of solution.

(ii) For each transmit channel, implement a numerical research to determine which difference vectors providing $d_{\text {min }}$

(iii) Equalize all difference distances of the vectors in step (ii) to obtain analytic solutions of the power allocation matrix $\boldsymbol{\Sigma}$.

In the case of the $4^{m}$-QAM modulation, we have $\left(2^{m+1}-1\right)^{2 b}$ difference vectors. Some of them cannot provide the minimum distance. It is due to the collinear properties, for example, $d_{\alpha \breve{\mathbf{x}}}^{2}=|\alpha|^{2} d_{\breve{\mathbf{x}}}^{2}>d_{\breve{\mathbf{x}}}^{2}$, with $\forall|\alpha|>$ 1 . For that reason, by eliminating all collinear difference vectors, we can reduce significantly the space of solutions.

In step (ii), we first parameterize the power allocation matrix $\Sigma$ as the form of some trigonometric elements, such as,

$$
\begin{array}{r}
\boldsymbol{\Sigma}=\sqrt{E_{s}} \operatorname{diag}\left(\cos \psi_{1}, \sin \psi_{1} \cos \psi_{2}, \ldots,\right. \\
\left.\sin \psi_{1} \sin \psi_{2} \ldots \sin \psi_{b}\right)
\end{array}
$$

For each channel $\mathbf{H}_{v}$, a numerical search over all angles $\psi_{i} \in(0, \pi)$ in order to maximize the minimum distance shows us which difference vectors providing the distance $d_{\text {min }}$. And finally, the analytic solution of the matrix $\Sigma$ can be derived by equalizing the difference distances given by these vectors. 


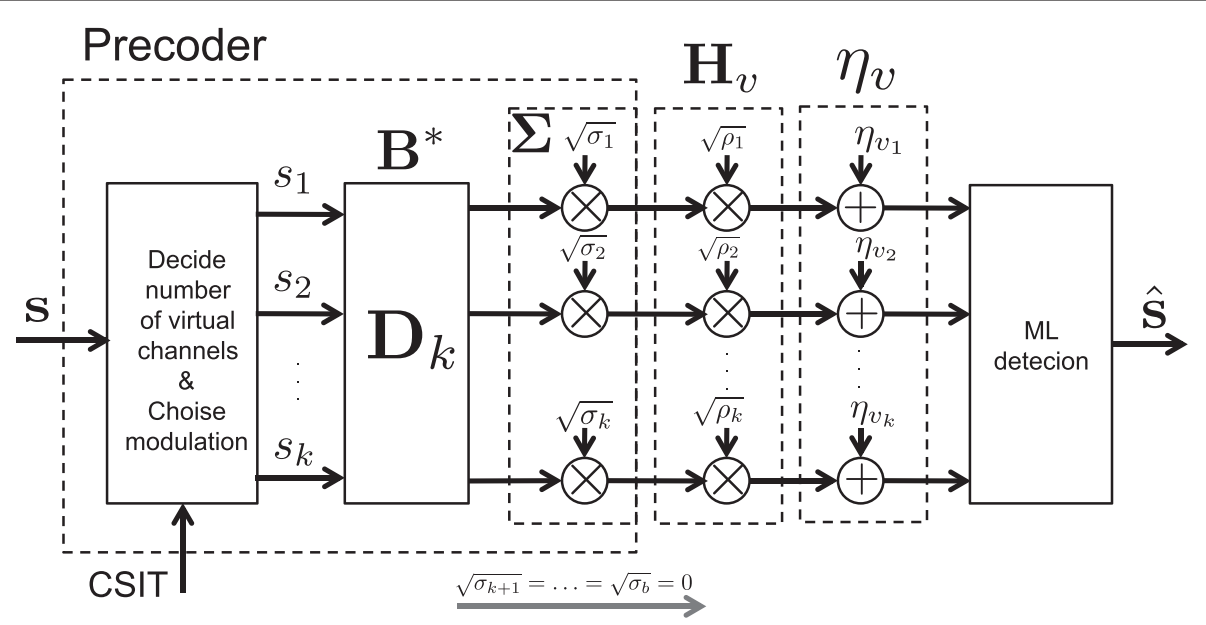

Figure 1 Design model of the precoding matrix.

One should note that the set of difference vectors, which provide $d_{\text {min }}$, is not fixed. It changes according to the channel values, and therefore lead to varies expressions of the matrix $\boldsymbol{\Sigma}$. For higher order QAM modulations, the form of our precoding matrix is more complicated. We propose, in this section, some characterized expressions of the new precoding scheme. These expressions are only suitable for the small dispersive channels (i.e., there is no much difference of SNRs between each subchannel). However, we can use them for all transmit channels because of their large gain in the performance of $d_{\mathrm{min}}$.

The number of non-null diagonal elements in $\Sigma$ represents the number of virtual-subchannels used for transmission. Let us denote the characterized expression of the precoder which enables powers on $k$ subchannels as $\mathbf{F}_{k}$ with $k=1, \ldots, b$. According to (25) and (27), the diagonal entries of the power allocation matrix $\Sigma$ can be defined by

$$
\sigma_{i}=\frac{E_{s}}{\sum_{j=1}^{k} \phi_{j} \rho_{j}^{-1}} \phi_{i} \rho_{i}^{-1}
$$

where $\phi_{j}$ denotes the power coefficient of the $j$ th virtual subchannel. It is obvious that the diagonal elements of $\Sigma$ are linearly proportional with $\phi_{j}$. We note that the value of $\phi_{j}$ depends on the set of the optimization vectors. By equalizing the difference distances obtained by these vectors, we can derive the analytic values of $\phi_{j}$. Some normalized coefficients $\phi_{j}$ are described in Table 1 . At the end of this section, we show how to obtain this coefficient and propose a method for the general case $\mathbf{F}_{k}$ (Table 1).

\section{Precoder $F_{1}$}

It is actually the max-SNR design which pours power on only the strongest virtual subchannel, i.e., $\boldsymbol{\Sigma}=$ $\operatorname{diag}\left\{\sqrt{E_{s}}, 0, \ldots, 0\right\}$. In order to retain the data-rate, the precoder $\mathbf{F}_{1}$ can use a higher-order QAM modulation. In other words, it can transforms $4^{m}$-QAM signals on $b$ virtual subchannels into a rectangular $4^{b . m}$-QAM signal on the first subchannel (detailed in Section "Range of definition"). The minimum distance provided by $\mathbf{F}_{1}$ is defined by

$$
d_{\mathbf{F}_{1}}^{2}=\frac{4}{M_{s}} E_{s} \rho_{1}
$$

\section{Precoder $F_{2}$}

This is the second expression of the $N-d_{\text {min }}$ precoder which is presented in our previous work [18]. We observe that the minimum distance is provided by two difference vectors $\breve{\mathbf{x}}_{1}=\frac{1}{\sqrt{M_{s}}}\left[\begin{array}{ll}0 & 2\end{array}\right]^{T}$, and $\breve{\mathbf{x}}_{2}=\frac{1}{\sqrt{M_{s}}}\left[\begin{array}{ll}2 & -2\end{array}\right]^{T}$. By substituting the DFT-matrix of size $b$ into (11), we have

$$
\mathbf{F}_{2}=\sqrt{\frac{E_{s}}{2}}\left(\begin{array}{cc}
\cos \psi & 0 \\
0 & \sin \psi
\end{array}\right)\left(\begin{array}{ll}
1 & 1 \\
-1 & 1
\end{array}\right) .
$$

The normalized distances provided by $\breve{\mathbf{x}}_{1}$ and $\breve{\mathbf{x}}_{2}$ are given by

$$
\left\{\begin{array}{l}
\bar{d}_{\breve{x}_{1}}^{2}=4 \rho_{1} \cos ^{2} \psi+4 \rho_{2} \sin ^{2} \psi \\
\bar{d}_{\breve{x}_{2}}^{2}=16 \rho_{2} \sin ^{2} \psi
\end{array}\right.
$$

Table 1 Optimized coefficients of the power allocation matrix $\Sigma$

\begin{tabular}{llllll}
\hline Expression & $\boldsymbol{\phi}_{\mathbf{1}}$ & $\boldsymbol{\phi}_{\mathbf{2}}$ & $\boldsymbol{\phi}_{\mathbf{3}}$ & $\boldsymbol{\phi}_{\mathbf{4}}$ & $\boldsymbol{\phi}_{\boldsymbol{k}}$ \\
\hline $\boldsymbol{\Sigma}_{1}$ & 1 & & & & \\
$\boldsymbol{\Sigma}_{2}$ & 3 & 1 & & & \\
$\boldsymbol{\Sigma}_{3}$ & $6+2 \sqrt{3}$ & $2+\sqrt{3}$ & 1 & & \\
$\boldsymbol{\Sigma}_{4}$ & 9 & 5 & 1 & 1 & \\
$\boldsymbol{\Sigma}_{k}$ & $\ldots$ & $\ldots$ & $\ldots$ & $\ldots$ & $\ldots$ \\
\hline
\end{tabular}


By equalizing $\bar{d}_{\breve{x}_{1}}^{2}=\bar{d}_{\breve{x}_{2}}^{2}$, we obtain $\psi=\operatorname{atan}\left(\sqrt{\rho_{1} / 3 \rho_{2}}\right)$. The distance $d_{\text {min }}$ provided by $\mathbf{F}_{2}$ is

$$
d_{\mathbf{F}_{2}}^{2}=\frac{4}{M_{s}} E_{s} \frac{2 \rho_{1} \rho_{2}}{\rho_{1}+3 \rho_{2}} .
$$

Figure 2 shows the received constellation provided by the precoder $\mathbf{F}_{2}$. It is observed that whenever two received vectors are close on one virtual subchannel, they are distant on the other (e.g., points A and B).

An exciting property of the precoding matrix $\mathbf{F}_{2}$ is that the average number of neighbors providing $d_{\min }$ is less than that of the optimized solution presented in [11]. For that reason, it provides a slight improvement in term of BER performance compared to the optimized $\max -d_{\min }$ precoder.

\section{Precoder $F_{3}$}

This precoder pours power on three virtual subchannels, and has a characterized expression which is defined by

$$
\mathbf{F}_{3}=\frac{1}{\sqrt{3}}\left(\begin{array}{ccc}
\sigma_{1} & 0 & 0 \\
0 & \sigma_{2} & 0 \\
0 & 0 & \sigma_{3}
\end{array}\right)\left(\begin{array}{ccc}
1 & 1 & 1 \\
1 & \frac{-1-\sqrt{3} i}{2} & \frac{-1+\sqrt{3} i}{2} \\
1 & \frac{-1+\sqrt{3} i}{2} & \frac{-1-\sqrt{3} i}{2}
\end{array}\right) \text {. }
$$

A numerical approach shows that the minimum distance is provided by three difference vectors $\breve{\mathbf{x}}_{1}=$ $\frac{1}{\sqrt{M_{s}}}[0,0,2]^{T}, \quad \breve{\mathbf{x}}_{2}=\frac{1}{\sqrt{M_{s}}}[0,2,-2]^{T}$, and $\breve{\mathbf{x}}_{3}=$ $\frac{1}{\sqrt{M_{s}}}[2,-2-2 i, 2 i]^{T}$. One should note that three difference vectors do not always give the distance $d_{\min }$. They are only available for small dispersive channel, i.e., $\rho_{1}$ is not too higher than $\rho_{2}$, and $\rho_{2}$ is not much higher than $\rho_{3}$. It is reasonable to choose these vectors, because when the channel is large dispersive $\left(\rho_{3} \gg \rho_{2}\right.$ or $\rho_{2} \gg \rho_{1}$, for example), we can use the precoding matrix $\mathbf{F}_{2}$ or $\mathbf{F}_{1}$.
By equalizing three difference distances provided by these vectors, we obtain

$$
\left\{\begin{array}{l}
\sigma_{2} / \sigma_{3}=\frac{2+\sqrt{3}}{\rho_{2} / \rho_{3}} \\
\sigma_{1} / \sigma_{3}=\frac{6+2 \sqrt{3}}{\rho_{1} / \rho_{3}}
\end{array}\right.
$$

The distance $d_{\min }$ obtained by $\mathbf{F}_{3}$ is then

$$
d_{\mathbf{F}_{3}}^{2}=\frac{4}{M_{s}} E_{s} \frac{(3+\sqrt{3}) \rho_{1} \rho_{2} \rho_{3}}{\rho_{1} \rho_{2}+(2+\sqrt{3}) \rho_{1} \rho_{3}+(6+2 \sqrt{3}) \rho_{2} \rho_{3}} .
$$

Figure 3 plots the received constellation provided by the precoder $\mathbf{F}_{3}$ in the case of 4-QAM. Like the case of the precoder $\mathbf{F}_{2}$, we observe that two received vectors processed by $\mathbf{F}_{3}$ are close on one virtual subchannel but can be distant on the others (e.g., points B and C).

\section{Precoder $F_{4}$}

The characterized expression of the precoding matrix $\mathbf{F}_{4}$ is given by

$$
\mathbf{F}_{4}=\frac{1}{2}\left(\begin{array}{cccc}
\sigma_{1} & 0 & 0 & 0 \\
0 & \sigma_{2} & 0 & 0 \\
0 & 0 & \sigma_{3} & 0 \\
0 & 0 & 0 & \sigma_{4}
\end{array}\right)\left(\begin{array}{cccc}
1 & 1 & 1 & 1 \\
1 & -i & -1 & i \\
1 & -1 & 1 & -1 \\
1 & i & -1 & -i
\end{array}\right)
$$

A numerical search shows that the minimum distance is provided by $\breve{\mathbf{x}}_{1}=\frac{1}{\sqrt{M_{s}}}[0,0,0,2]^{T}, \breve{\mathbf{x}}_{2}=$ $\frac{1}{\sqrt{M_{s}}}[0,0,2,-2]^{T}, \breve{\mathbf{x}}_{3}=\frac{1}{\sqrt{M_{s}}}[0,2,-2-2 i, 2 i]^{T}$, and
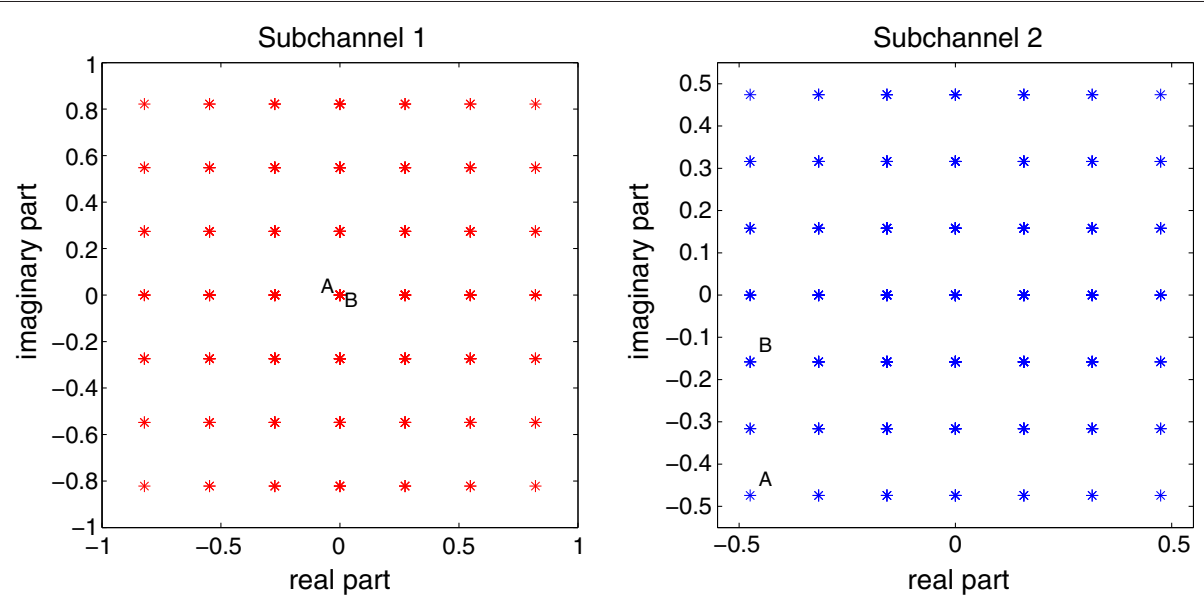

Figure 2 Received constellations provided by the precoder $\mathbf{F}_{\mathbf{2}}$. 

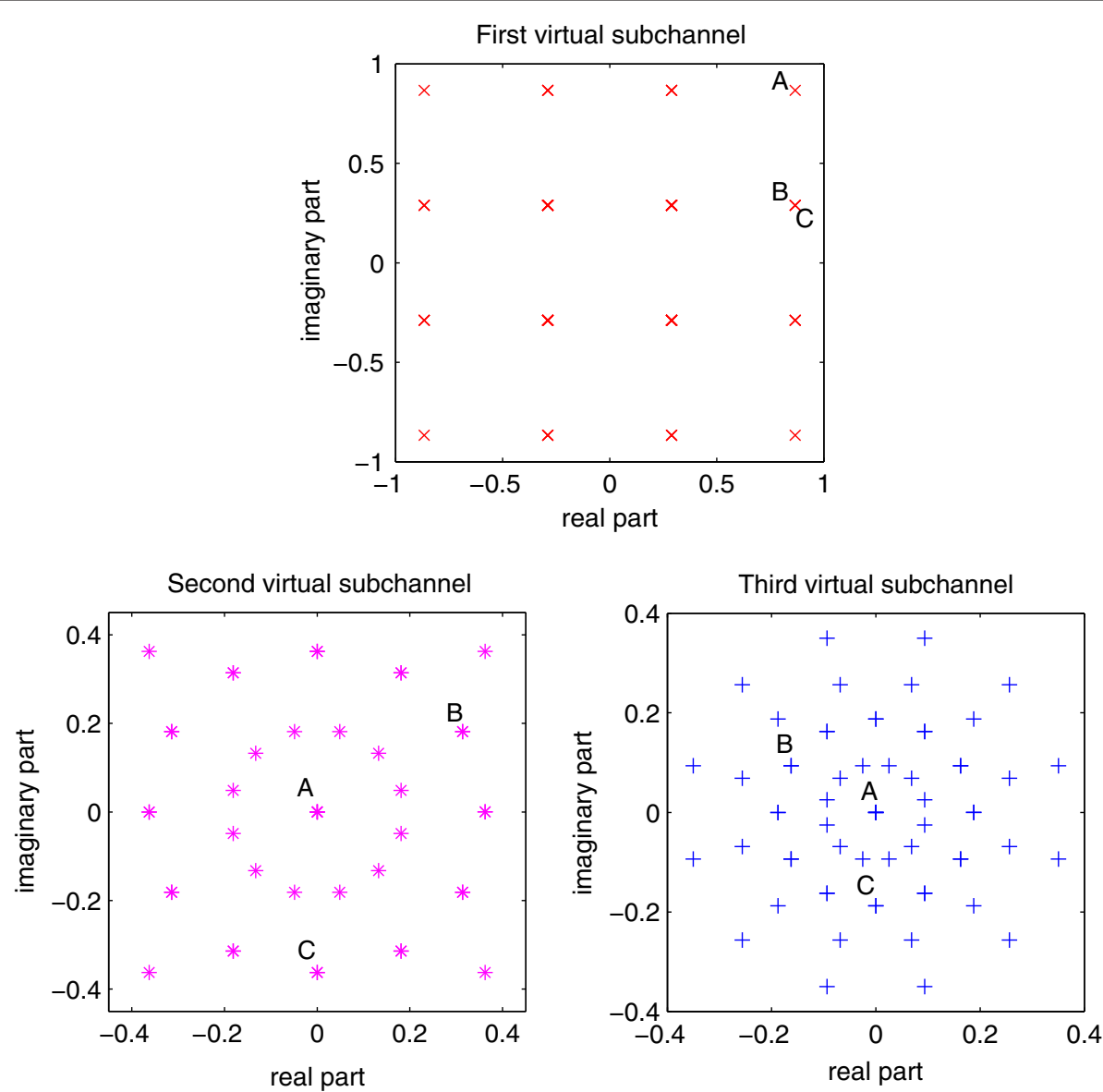

Figure 3 Received constellations provided by the precoder $\mathbf{F}_{\mathbf{3}}$.

$\breve{\mathbf{x}}_{4}=\frac{1}{\sqrt{M_{s}}}[2,-2,2,-2]^{T}$. Like the case of the precoder $\mathbf{F}_{3}$, by equalizing their difference distances, we obtain

$$
\left\{\begin{array}{l}
\sigma_{3} / \sigma_{4}=\frac{1}{\rho_{3} / \rho_{4}} \\
\sigma_{2} / \sigma_{4}=\frac{5}{\rho_{2} / \rho_{4}} \\
\sigma_{1} / \sigma_{4}=\frac{9}{\rho_{1} / \rho_{4}}
\end{array}\right.
$$

The distance $d_{\text {min }}$ obtained by $\mathbf{F}_{4}$ is given by

$$
d_{\mathbf{F}_{4}}^{2}=\frac{4}{M_{s}} E_{s} \frac{4}{9 / \rho_{1}+5 / \rho_{2}+1 / \rho_{3}+1 / \rho_{4}} .
$$

\section{The general case $F_{k}$}

In the case of $k$ parallel datastreams, a first numerical approach is first consider to determine all optimization vectors. This numerical search is implemented for all angles $\psi_{i} \in(0, \pi)$ of the matrix $\boldsymbol{\Sigma}$ in (29) to optimize the distance $d_{\text {min }}$. Let us denote $\breve{\mathbf{x}}_{1}, \breve{\mathbf{x}}_{2}, \ldots, \breve{\mathbf{x}}_{k}$ as $k$ difference vectors providing the minimum distance. The distance $d_{\breve{\mathbf{x}}_{i}}$ is given by

$$
\begin{aligned}
& d_{\breve{\mathbf{x}}_{i}}^{2}=\breve{\mathbf{x}}_{i}^{*} \mathbf{B} \Upsilon \mathbf{B}^{*} \breve{\mathbf{x}}_{i} \\
& =\sum_{j=1}^{k} \lambda_{j}\left|u_{i(j)}\right|^{2}
\end{aligned}
$$

where $\boldsymbol{\Upsilon}=\boldsymbol{\Sigma}^{*} \mathbf{H}_{v}^{*} \mathbf{H}_{v} \boldsymbol{\Sigma}=\operatorname{diag}\left(\lambda_{1}, \ldots, \lambda_{k}\right)$, and vector $\mathbf{u}_{i}$ is given by

$$
\mathbf{u}_{i}=\mathbf{B}^{*} \breve{\mathbf{x}}_{i}=\left[u_{i(1)}, u_{i(2)}, \ldots, u_{i(k)}\right]^{T} .
$$

By equalizing $k$ difference distances, we have $(k-1)$ equations below

$$
\sum_{j=1}^{k} \lambda_{j}\left(\left|u_{1(j)}\right|^{2}-\left|u_{i(j)}\right|^{2}\right)=\sum_{j=1}^{k} \lambda_{j} v_{i, j}=0,
$$

where $v_{i, j}=\left|u_{1(j)}\right|^{2}-\left|u_{i(j)}\right|^{2}$ with $i=2, \ldots, k$. For a $4^{m_{-}}$ QAM modulation, it is noted that the difference vector $\breve{\mathbf{x}}_{1}$ is often defined by $\breve{\mathbf{x}}_{1}=[0, \ldots, 0,2]^{T}$, i.e., $\left|u_{1(j)}\right|^{2}=4$ 
with $j=1 \ldots k$. The power constrain in (12) can be now rewritten as

$$
\sum_{j=1}^{k} \lambda_{j} / \rho_{j}=\sum_{j=1}^{k} \sigma_{j}=E_{s}
$$

Let us define $\lambda=\left[\lambda_{1}, \ldots, \lambda_{k}\right]^{T}$, and $v_{1, j}=1 / \rho_{j}$ with $j=$ $1, \ldots, k$, we have

$$
\left(\begin{array}{cccc}
v_{1,1} & v_{1,2} & \ldots & v_{1, k} \\
v_{2,1} & v_{2,2} & \ldots & v_{2, k} \\
\vdots & \vdots & & \vdots \\
v_{k, 1} & v_{k, 2} & \ldots & v_{k, k}
\end{array}\right)\left(\begin{array}{c}
\lambda_{1} \\
\lambda_{2} \\
\vdots \\
\lambda_{k}
\end{array}\right)=\left(\begin{array}{c}
E_{s} \\
0 \\
\vdots \\
0
\end{array}\right)
$$

or

$$
\mathrm{V} \lambda=\epsilon .
$$

In conclusion, the power coefficients $\phi_{i}$ are proportional to the entries of the vector $\lambda$ which can be defined by $\lambda=$ $\mathbf{V}^{-1} \boldsymbol{\epsilon}$. The condition of the existence of the vector $\lambda$ is that the matrix $\mathbf{V}$ is invertible. When $\breve{\mathbf{x}}_{1}=[0, \ldots, 0,2]^{T}$ is one of the difference vectors providing the minimum distance, the distance $d_{\min }$ is then defined by

$$
d_{\mathbf{F}_{k}}^{2}=4 \sum_{j=1}^{k} \lambda_{j}
$$

\section{Range of definition}

To improve the BER performance of a MIMO system, we choose from these precoding matrices above the precoder that provides the highest minimum Euclidean distance. One should note that the data-rate of a precoder $\mathbf{F}_{i}$ is different to each other's. For example, if we both use 4QAM modulation for the precoders $\mathbf{F}_{1}$ and $\mathbf{F}_{2}$, the bit-rate of $\mathbf{F}_{2}$ is twice as that of $\mathbf{F}_{1}$. Therefore, we have to consider the data-rate of the $b$ precoders when comparing their distances $d_{\text {min }}$. The error probability in (7) can be re-expressed as

$$
P_{e} \approx N_{d_{\min }} Q\left(\frac{\bar{d}_{\text {min }}}{2} \times \sqrt{\operatorname{SNR} \frac{B}{f_{s}} \frac{1}{\log _{2} M}}\right),
$$

where $M$ is the number of alternative modulation symbols, $B$ is the bandwidth, and $f_{s}$ is the symbol rate. For a given modulation order, by comparing the right-hand side of (48) corresponding to $b$ precoders, we can obtain the range of definition for each precoding scheme.

Another simple method to retain the data-rate is using different modulation for each precoder. Lets us come back to the example of the precoders $\mathbf{F}_{1}$ and $\mathbf{F}_{2}$. If the 4-QAM modulation is used for the precoder $\mathbf{F}_{2}$, it means that two 2-bits symbols are transferred on two subchannels. Instead of transmitting like this, we can transfer one 4bits symbols (16-QAM) on the first virtual subchannels. Then, two minimum distances that correspond to $\mathbf{F}_{1}$ using 16-QAM and $\mathbf{F}_{2}$ using 4-QAM are compared in order to determine the range of definition for two precoders $\mathbf{F}_{1}$ and $\mathbf{F}_{2}$.

$$
\left\{\begin{array}{l}
d_{\mathbf{F}_{1}}^{2}=\frac{2}{5} E_{s} \rho_{1} \\
d_{\mathbf{F}_{2}}^{2}=2 E_{s} \frac{2 \rho_{1} \rho_{2}}{\rho_{1}+3 \rho_{2}}
\end{array}\right.
$$

In other words, if $d_{\mathbf{F}_{1}}^{2}>d_{\mathbf{F}_{2}}^{2}$ or $\rho_{1} / \rho_{2}>7$ : the precoder $\mathbf{F}_{1}$ is chosen, and for $\rho_{1} / \rho_{2}<7$ : the precoder $\mathbf{F}_{2}$ is selected. Other precoders can be implemented in a similar way.

\section{Simulation results}

\section{Comparison of minimum Euclidean distance}

In this section, we indicate the improvement of the proposed precoder in terms of the minimum Euclidean distance compared to diagonal precoders. Indeed, the minimum Euclidean distance provided by a diagonal precoder is

$$
\begin{aligned}
d_{\min }^{2} & =\min _{\mathbf{s}, \mathbf{r} \in S, \mathbf{s} \neq \mathbf{r}}\left\|\mathbf{H}_{v} \mathbf{F}_{d}(\mathbf{s}-\mathbf{r})\right\|^{2} \\
& =\min _{\mathbf{s}, \mathbf{r} \in S, \mathbf{s} \neq \mathbf{r}} \sum_{i}^{b} \rho_{i} f_{i}^{2}\left|s_{i}-r_{i}\right|^{2}
\end{aligned}
$$

where $\mathbf{s}=\left[s_{1}, s_{2}, \ldots, s_{b}\right]^{T}, \mathbf{r}=\left[r_{1}, r_{2}, \ldots, r_{b}\right]^{T}$, and $\mathbf{F}_{d}=\operatorname{diag}\left(f_{1}, \ldots, f_{b}\right)$. One should note that the minimum Euclidean distance is obtained when the two vectors $\mathbf{s}$ and $\mathbf{r}$ are different from only a symbol. The minimum Euclidean distance of $\mathbf{F}_{d}$ is then given by

$$
\begin{aligned}
d_{\min }^{2} & =\min _{\mathbf{s}, \mathbf{r} \in S, \mathbf{s} \neq \mathbf{r}} \min _{i=1 \ldots b} \rho_{i} f_{i}^{2}\left|s_{i}-r_{i}\right|^{2} \\
& =\min _{i=1 \ldots b} \rho_{i} f_{i}^{2} \min _{\mathbf{s}, \mathbf{r} \in S, \mathbf{s} \neq \mathbf{r}}\left|s_{i}-r_{i}\right|^{2} \\
& =\frac{4}{M_{s}} \min _{i=1 \ldots b} \rho_{i} f_{i}^{2} .
\end{aligned}
$$

It is noted that the diagonal entries of $\mathbf{H}_{v}=$ $\operatorname{diag}\left(\sqrt{\rho_{1}}, \ldots, \sqrt{\rho_{b}}\right)$ are sorted in decreasing order, i.e., $\rho_{1} \geq \rho_{2} \geq \cdots \geq \rho_{b}$. By comparing all of elements on right-hand side of (51), the minimum distances corresponding to some traditional precoders such as beamforming, $\max -\lambda_{\min }$ [8], Water-filling [4], and MMSE [7], can be determined. Table 2 illutrates the distance $d_{\min }$ obtained by these diagonal precoders in comparison with our proposed precoder, in which $(x)^{+} \stackrel{\text { def }}{=} \max (x, 0)$.

The normalized minimum distances provided by these precoder above, in the case of $b=2$ virtual subchannels and 4-QAM modulation, are illustrated in Figure 4. It is observed that our precoder provides a large improvement in terms of $d_{\text {min }}$ compared to the diagonal precoders. In comparison with the $\max -d_{\text {min }}$ precoder presented in [11], the proposed precoder has a small difference in the minimum distance. However, its average number of neighbors providing the minimum distance is less than that of 
Table 2 Comparison of the minimum Euclidean distances

\begin{tabular}{|c|c|}
\hline Precoder & Minimum Euclidean distance $d_{\min }^{2}$ \\
\hline Beamforming & $\frac{4}{M_{s}} E_{S} \rho_{1}$ \\
\hline Water-filling & $\frac{4}{M_{s}}\left(\rho_{b} \frac{E_{s}+\sum_{j=1}^{b} 1 / \rho_{j}}{b}-1\right)^{+}$ \\
\hline MMSE & $\frac{4}{M_{s}}\left(\sqrt{\rho_{b}} \frac{E_{s}+\sum_{j=1}^{b} 1 / \rho_{j}}{\sum_{j=1}^{b} 1 / \sqrt{\rho_{j}}}-1\right)^{+}$ \\
\hline $\max -\lambda \min$ & $\begin{array}{l}\frac{4}{M_{s}} \frac{E_{s}}{\sum_{j=1}^{b} \frac{1 / \rho_{j}}{M_{s}}} \\
\quad \int \frac{4}{M_{s} \rho_{1}}\end{array}$ \\
\hline Our proposed & $\left\{\begin{array}{l}\frac{4}{M_{s}} E_{s} \frac{2 \rho_{1} \rho_{2}}{\rho_{1}+3 \rho_{2}} \\
\ldots \ldots \ldots\end{array}\right.$ for $\mathbf{F}_{2}$ \\
\hline
\end{tabular}

the $\max -d_{\min }$ precoder [18]. According to that improvement, an enhancement in terms of BER is expected for our new precoder (Table 2).

\section{BER performance}

In this section, the BER performance of the proposed precoder is considered in comparison with other traditional precoding strategies. The proposed precoder obtains a significant improvement of BER in comparison with the diagonal precoders: Water-filling, MMSE, and $\max -\lambda_{\min }$. A gain about $6 \mathrm{~dB}$ can be observed at high SNR. Furthermore, as discussed above, our precoder has the number of neighbors providing $d_{\min }$ less than that of the optimal solution $\max -d_{\min }$ in [11], although it has a small difference in terms of $d_{\text {min }}$. Therefore, the new precoder provides a slight BER improvement compared to the $\max -d_{\text {min }}$ solution. The BER performance with respect to SNR for two transmit datastreams and 4-QAM modulation is plotted in Figure 5.

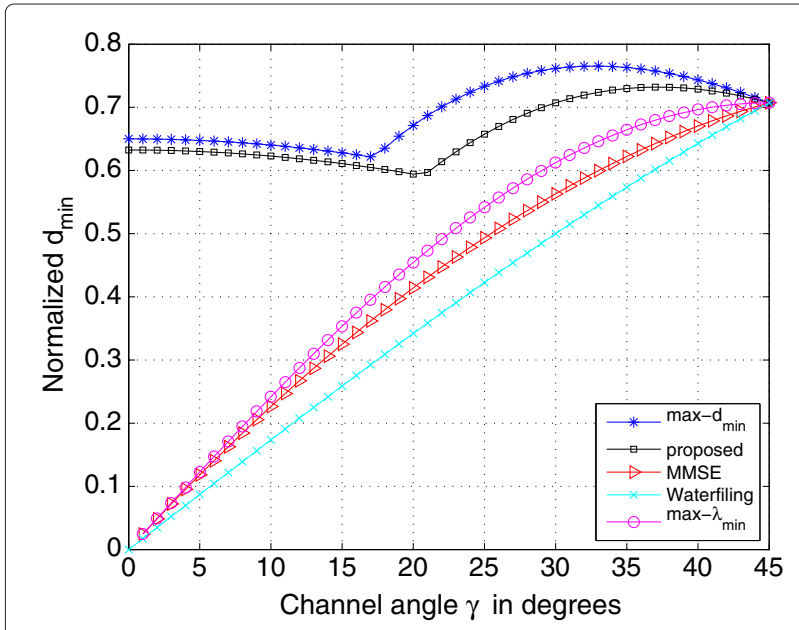

Figure 4 Normalized minimum Euclidean distance for two datastreams and 4-QAM modulation, with the channel angle $\gamma=\operatorname{atan} \sqrt{\rho_{2} / \rho_{1}}$.
The optimal solution for $\max -d_{\min }$ precoder is presented in $[11,12]$, but it is only available for two transmit datastreams with 4-QAM and 16-QAM modulations. By decomposing the channel into $2 \times 2$ eigen-channel matrices and optimize the distance $d_{\text {min }}$ for each pair of datastreams, Vrigneau et al. [13] proposed a sub-optimal precoder for large MIMO channels. This extension is split into four steps: virtual diagonalization of the channel, combination in pairs of sub-channels, application of the optimal 2D max $-d_{\min }$ solution, and power allocation on each sub-system. However, this solution is also suitable for low-order QAM modulations. A main advantage of our new precoder is that the solution is available for all rectangular QAM-modulations and for any number of datastreams.

For large MIMO simulations, we consider a system with $n_{T}=5$ transmit and $n_{R}=4$ receive antennas. The bitstreams are separated into $b=4$ independent virtual subchannels, and the channel matrix $\mathbf{H}$ is i.i.d. zero-mean complex Gaussian. For each SNR, the precoders are optimized for about 30,000 random matrices $\mathbf{H}$. It is observed in Figure 5 that the BER performance of the $\max -\lambda_{\min }$ solution is better than those of MMSE and Water-filling. Therefore, the $\max -\lambda_{\min }$ precoder is chosen to compare with our proposed precoder. Beside that some sophisticated transceivers such as: the Schur-convex ARITH-BER design [9], the linear precoder using Decision Feedback Equalization (DFE) [19], and the linear transceiver with bit allocation [20] are also mentioned in the comparison with our precoder. Figure 6 illustrates the BER performance for MIMO $(5,4)$ systems using 4-QAM modulation. The comparison of the proposed precoder and other schemes for $b=4$ transmit datastreams shows that the performance is significantly enhanced in terms of BER. It is obvious that our precoder performs much better than diagonal

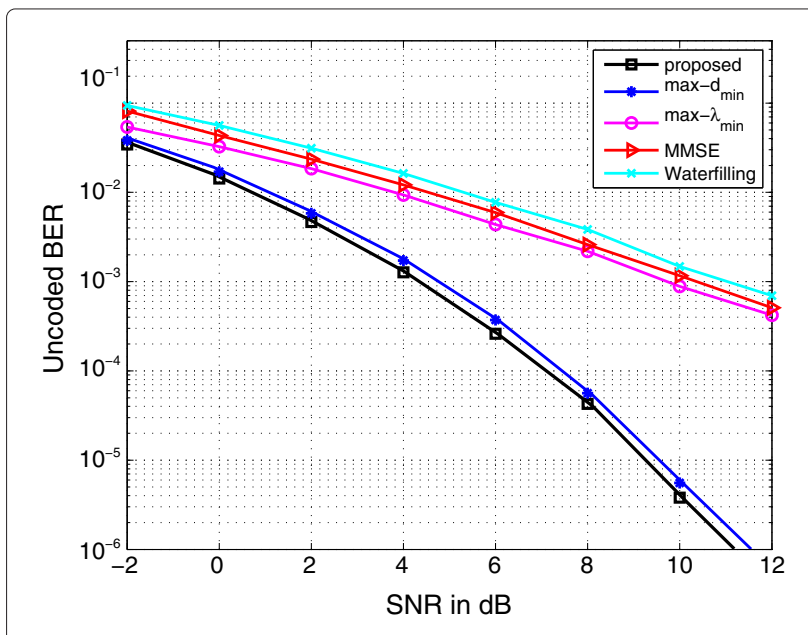

Figure 5 Uncoded BER performance for $b=2$ datastreams. 


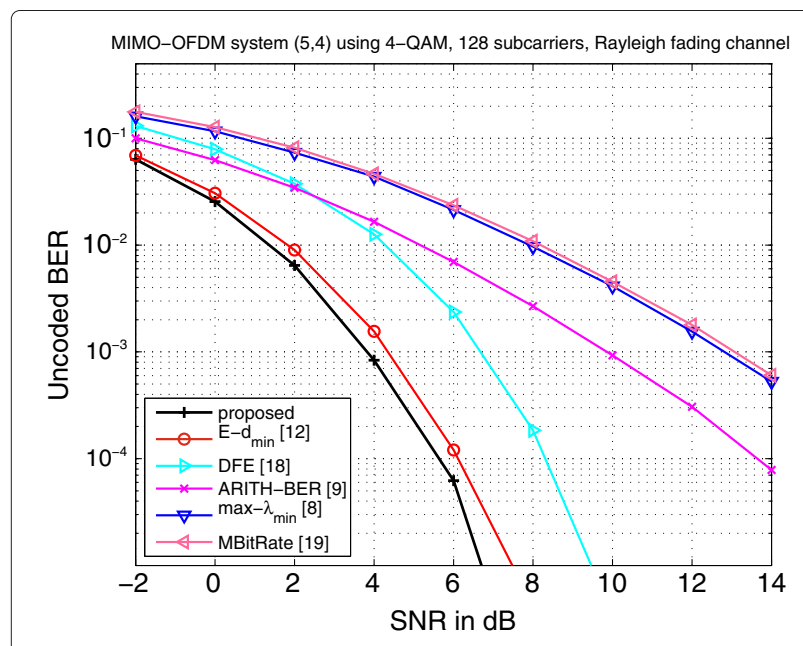

Figure 6 Comparison of BER performance for large MIMO(5,4) systems.

precoders such as $\max -\lambda_{\min }$ and Water-filling. Furthermore, we observe that the new precoder also presents a significant improvement of BER compared to the DFE, the Schur-convex ARITH-BER, and the maximum bit-rate solutions, especially when the SNR is high. The new precoder was found to be better than $E-d_{\min }$ schemes and this is due to the fact that not only the minimum distance, but also the number of neighbors providing $d_{\min }$ is taken into consideration.

We also consider, in this section, the impact of imperfect CSI estimation on the BER performance of the proposed precoder. Figure 7 illustrates the BER performance with respect to SNR in the case of perfect CSI and imperfect CSI estimation. The estimated channel matrix of imperfect CSI system can be modeled as $\mathbf{H}_{\mathrm{est}}=\mathbf{H}+\mathbf{H}_{\mathrm{err}}$, where

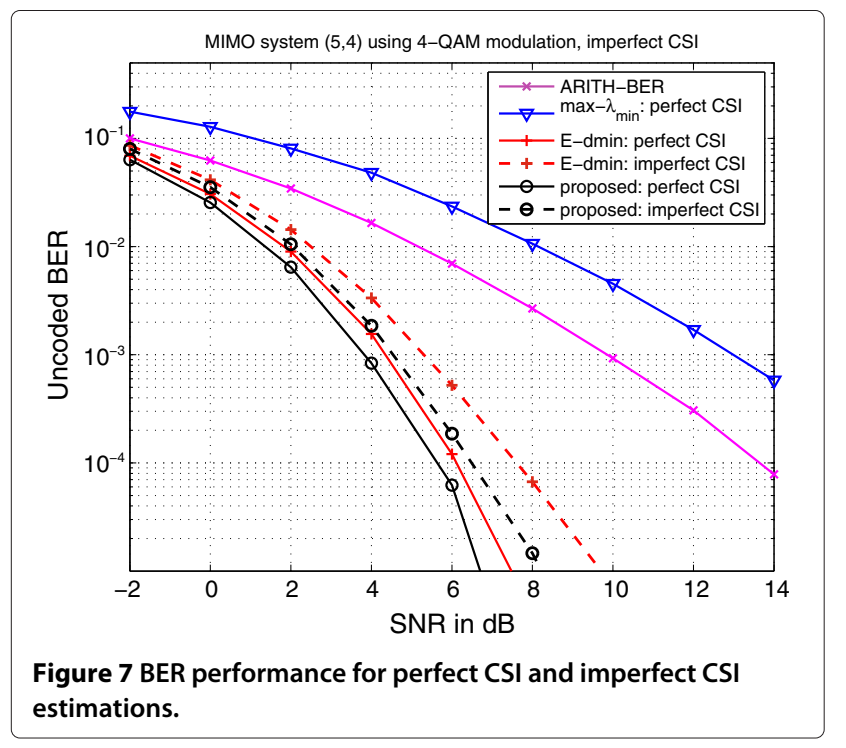

$\mathbf{H}_{\text {err }}$ represents the channel estimation error. The optimal training signals for the MIMO-OFDM channel estimation can be found in [21]. One should note that if the transmit channel is quasi-stationary, i.e., remains constant for several symbol periods, the precoding across time could be replaced by precoding across subcarriers [14]. In this simulation, we assume that the entries of $\mathbf{H}_{\mathrm{err}}$ are complex Gaussian i.i.d random with mean zero and variance $\sigma_{\text {err }}=0.3 \sigma$, where $\sigma$ is the variance of the complex Gaussian entries of $\mathbf{H}$. It is observed that the BER performance of our precoder decreases at high SNR, but it still remains better than the other precoding strategies. Furthermore, the BER enhancement obtained by the proposed precoder is much better than the case of full CSI in comparison with the $\mathrm{E}-d_{\min }$ solution: a gain of $2 \mathrm{~dB}$ in SNR can be observed at $\mathrm{BER}=10^{-5}$.

\section{Conclusion}

We proposed, in this article, a new linear precoder that is based on the maximization of the minimum Euclidean distance between two received data vectors. The suboptimal design was obtained by observing the SNR-like matrix of the precoding matrix. An approximation of the minimum distance is derived, and its maximum value was obtained by maximizing the minimum diagonal element of the SNR-like matrix. We then showed that the minimum diagonal element can be attained by a specific set of the precoder. Firstly, the precoding matrix is parameterized as the product of a diagonal power allocation matrix and an input-shaping matrix. The input-shaping matrix concerns with the rotation and scaling of the input symbols on each virtual subchannel. We demonstrated that the minimum diagonal entry of the SNR-like matrix is obtained from a special choice of the input-shaping matrix, i.e., a DFT-matrix, and our objective becomes determining the power allocation matrix $\boldsymbol{\Sigma}$. As its name implies, the matrix $\boldsymbol{\Sigma}$ decides how many subchannels are used by the precoder for data transmission. For each number of available datastreams, we proposed a simple characterized expression of the precoding matrix for all rectangular QAM modulations.

We also presented some performance comparisons to demonstrate that the proposed precoder obtains a significant improvement in terms of BER. The improvement may be more than several $d B$ at reasonable BER levels. In comparison with the optimal $\max -d_{\min }$ solution, our proposed precoder also provides a slight BER improvement. One main advantage of our design is that the solution can be available for all rectangular QAMmodulations and for any number of datastreams. It is because that the precoder has a simple analytic form, and the space of the solution is smaller than the full design of minimum distance based precoders. 


\section{Competing interests}

The authors declare that they have no competing interests.

Received: 1 August 2011 Accepted: 23 November 2012

Published: 2 March 2013

\section{References}

1. A Paulraj, D Gore, R Nabar, H Bolcskei, An overview of MIMO communications-a key to gigabit wireless. Proc IEEE. 92(2), 198-218 (2004)

2. G Foschini, Layered space-time architecture for wireless communication in a fading environment when using multi-element antennas. Bell Labs. Tech J. 1(2), 41-59 (1996)

3. $\mathrm{M} \mathrm{Vu}, \mathrm{A}$ Paulraj, MIMO wireless linear precoding. IEEE Signal Process Mag 24(5), 86-105 (2007)

4. E Telatar, Capacity of multi-antenna Gaussian channels. Eur. Trans Telecommun. 10(6), 585-595 (1999)

5. P Rostaing, O Berder, G Burel, L Collin, Minimum BER diagonal precoder for MIMO digital transmissions. IEEE Signal Process. 82(10), 1477-1480 (2002)

6. P Stoica, G Ganesan, Maximum-SNR spatial-temporal formatting designs for MIMO channels. IEEE Trans. Signal Process. 50(12), 3036-3042 (2002)

7. H Sampath, P Stoica, A Paulraj, Generalized linear precoder and decoder design for MIMO channels using the weighted MMSE criterion. IEEE Trans Commun. 49(12), 2198-2206 (2001)

8. A Scaglione, P Stoica, S Barbarossa, G Giannakis, H Sampath, Optimal designs for space-time linear precoders and decoders. IEEE Trans. Signal Process. 50(5), 1051-1064 (2002)

9. D Palomar, J Cioffi, M Lagunas, Joint tx-rx beamforming design for multicarrier mimo channels: a unified framework for convex optimization. IEEE Trans. Signal Process. 51(9), 2381-2401 (2003)

10. F Perez-Cruz, MRD Rodrigues, S Verdu, MIMO Gaussian channels with arbitrary inputs: optimal precoding and power allocation. IEEE Trans. Inf. Theory. 56(3), 1070-1084 (2010)

11. L Collin, O Berder, P Rostaing, G Burel, Optimal minimum distance-based precoder for MIMO spatial multiplexing systems. IEEE Trans. Signal Process. 52(3), 617-627 (2004)

12. Q-T Ngo, O Berder, B Vrigneau, O Sentieys, in IEEE International Conference on Communications (ICC). Minimum distance based precoder for mimo-ofdm systems using 16-qam modulation, (Dresden, Germany, June 2009)

13. B Vrigneau, J Letessier, P Rostaing, L Collin, G Burel, Extension of the MIMO precoder based on the minimum Euclidean distance: a cross-form matrix. IEEE J. Sel. Topics Signal Process. 2(2), 135-146 (2008)

14. D Kapetanović, F Rusek, in IEEE International Conference on Communications (ICC). On precoder design under maximum-likelihood detection for quasi-stationary mimo channels, (Cape Town, South Africa, May 2010)

15. S Bergman, D Palomar, B Ottersten, Joint bit allocation and precoding for mimo systems with decision feedback detection. IEEE Trans. Signal Process. 57(11), 4509-4521 (2009)

16. M Ma, X Huang, B Jiao, Y Guo, Optimal orthogonal precoding for power leakage suppression in dft-based systems. IEEE Trans. Commun. 59(3), 844-853 (2011)

17. A Goldsmith, Wireless Communications. (Cambridge University Press, Cambridge, 2005)

18. Q-T Ngo, O Berder, P Scalart, in IEEE Wireless Communications and Networking Conference (WCNC). Reducing the number of neighbors in the received constellation of dmin precoded mimo systems, (Cancun, Mehico, March 2011)

19. M Shenouda, T Davidson, A framework for designing mimo systems with decision feedback equalization or tomlinson-harashima precoding. IEEE J. Sel. Areas Commun. 26(2), 401-411 (2008)

20. C Li, Y Lin, S Tsai, P Vaidyanathan, Optimization of transceivers with bit allocation to maximize bit rate for mimo transmission. IEEE Trans Commun. 57(12), 3556-3560 (2009)

21. H Minn, N Al-Dhahir, Optimal training signals for MIMO OFDM channel estimation. IEEE Trans. Wirel. Commun. 5(5), 1158-1168 (2006)

doi:10.1186/1687-6180-2013-39

Cite this article as: Ngo et al: General minimum Euclidean distance-based precoder for MIMO wireless systems. EURASIP Journal on Advances in Signal Processing 2013 2013:39.

\section{Submit your manuscript to a SpringerOpen ${ }^{\circ}$ journal and benefit from:}

- Convenient online submission

- Rigorous peer review

- Immediate publication on acceptance

- Open access: articles freely available online

- High visibility within the field

- Retaining the copyright to your article

Submit your next manuscript at $\boldsymbol{\wedge}$ springeropen.com 